\title{
Features of the electronic structure of FeTe compounds
}

Cite as: Low Temp. Phys. 41, 990 (2015); https://doi.org/10.1063/1.4938519

Published Online: 31 December 2015

G. E. Grechnev, A. A. Lyogenkaya, A. S. Panfilov, A. V. Logosha, O. V. Kotlyar, V. P. Gnezdilov, I. P. Makarova,

D. A. Chareev, and E. S. Mitrofanova

\section{ARTICLES YOU MAY BE INTERESTED IN}

Superconductivity in S-substituted FeTe

Applied Physics Letters 94, 012503 (2009); https://doi.org/10.1063/1.3058720

Superconducting, magnetic and magnetotransport properties of $\mathrm{FeTe}_{1-\chi} \mathrm{Se}_{\mathrm{X}}$ single crystals AIP Advances 8, 055819 (2018); https://doi.org/10.1063/1.5007291

Pseudogap behaviour in FeTe and FeSe probed by photoemission AIP Conference Proceedings 1665, 130015 (2015); https://doi.org/10.1063/1.4918163

LOW TEMPERATURE TECHNIQUES OP『]CAL CAVITY PHYSICS MITIGATING THERMAL \& VIBRATIONAL NOISE

DOWNLOAD THE WHITE PAPER 


\title{
Features of the electronic structure of FeTe compounds
}

\author{
G. E. Grechnev, ${ }^{\text {a) }}$ A. A. Lyogenkaya, A. S. Panfilov, A. V. Logosha, O. V. Kotlyar, and \\ V. P. Gnezdilov
}

B. Verkin Physics and Technology Institute for Low Temperatures, NAS Ukraine, 47 Lenin Ave., Kharkov 61103, Ukraine

\section{P. Makarova}

Shubnikov Institute of Crystallography, Russian Academy of Sciences, Moscow 119333, Russia

\section{A. Chareev}

Institute of Experimental Mineralogy, Russian Academy of Sciences, Chernogolovka 142432, Russia

\section{E. S. Mitrofanova}

Physics Faculty, Lomonosov Moscow State University, Moscow 119991, Russia

(Submitted June 10, 2015; corrected 3 February 2016)

Fiz. Nizk. Temp. 41, 1268-1275 (December 2015)

\begin{abstract}
A theoretical and experimental study of the electronic structure and nature of the chemical bonds in FeTe compounds in antiferromagnetic (AFM) and paramagnetic phases was carried out. It is established that the nature of the chemical bonds is mainly metallic, and the presence of covalent bonds Fe-Te and Te-Te helps to stabilize the structural distortions of the tetragonal phase of FeTe in the low-temperature region. It is found that the bicollinear AFM structure corresponds to the ground state of the FeTe compound and the calculated value of the magnetic moment $M_{F e}=-2.4 \mu_{B}$ is in good agreement with the data from neutron diffraction measurements. At the same time, the Fermi surface (FS) of the low-temperature AFM phase is radically different from the FS of the paramagnetic FeTe. Reconstructing the FS can lead to a sign change of the Hall coefficient observed in FeTe. The calculation results serve as evidence of the fact that the electronic structures and magnetic properties of FeTe are well-described by the model of itinerant $d$-electrons and the density functional theory (DFT-GGA). (C) 2015 AIP Publishing LLC. [http://dx.doi.org/10.1063/1.4938519]
\end{abstract}

\section{Introduction}

The recent discovery of iron-based superconductors (SC) $\mathrm{FeSe}_{1-x} \mathrm{Te}_{x}$ has attracted special attention from researchers due to the simplicity of their crystalline structure. For these isovalent iron chalcogenides, the preservation of the anisotropic tetragonal structure like $\mathrm{PbO}$ is typical for a wide range of concentrations, which favors a detailed study of the effects of chemical substitution $\mathrm{Se} \Longleftrightarrow \mathrm{Te},{ }^{1-6}$ and high pressure, ${ }^{7}$ with the goal of determining the mechanism responsible for superconductivity.

The boundary compounds of the indicated solid solutions FeSe and FeTe, have similar electronic structures with a typical "nesting" of the Fermi Surface (FS), which means there are electron and hole corrugated cylinders embedded in the FS, in the paramagnetic phase. ${ }^{8}$ This nesting can contribute to the implementation of both the band antiferromagnetism (AFM) and the proposed non-traditional mechanism of superconductivity which occurs via AFM spin fluctuations with the nesting vector $\mathbf{Q}$, which corresponds to the spin-density wave. ${ }^{8,9}$ For iron pnictides, there is similar FS nesting, which leads to typical AFM ordering of Fe moments in the basal plane in stoichiometric compounds (such as $\mathrm{LaFeAsO}$ ), in the form of alternating collinear bands ("stripes," "single-stripe") with $\mathbf{Q}=(\pi, \pi)$. It was assumed that FeTe could also be a superconductor, similar to FeSe, due to its nesting. ${ }^{8,9}$ However, in samples $\mathrm{Fe}_{1+y} \mathrm{Te}$ at temperatures below $70 \mathrm{~K}$, there was an AFM state with a unique bicollinear stripe structure (“double-stripe," see Fig. 1), and a spin-density propagation vector $\mathbf{Q}=(\pi, 0) .{ }^{10-12}$

The observed particularity along the temperature dependence of magnetic susceptibility $\chi(T)$ at $T \simeq 70 \mathrm{~K}$ (Refs. 13 and 14) is not typical for the transition into the AFM phase, and is associated with the simultaneous first-order structural phase transition from a tetragonal crystal structure to the low-temperature monoclinic phase, which is accompanied by the appearance of antiferromagnetism. ${ }^{3,10,11}$ For $\mathrm{Fe}_{1+y} \mathrm{Te}$ compounds that are almost stoichiometric, at low temperatures, the monoclinic crystal structure and AFM ordering in the basal plane $a b$, are realized. ${ }^{3,11}$ The nature of the pronounced correlation between the structural and magnetic transitions in $\mathrm{Fe}_{1+y} \mathrm{Te}$ is not clear. It was assumed ${ }^{10}$

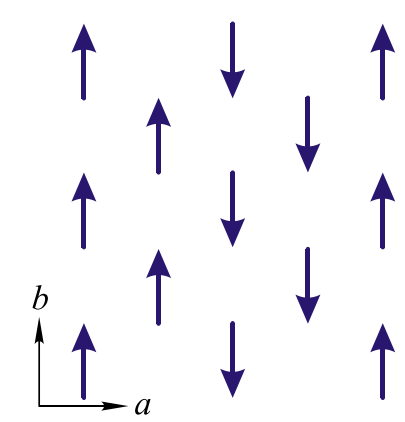

FIG. 1. A diagram of the ordering of the magnetic moments of iron ions in the basal plane of the FeTe compound, in a bicollinear AFM phase. 
that the main input to the change in entropy during the transition comes from AFM ordering, and that the transition itself is due to the magnetic subsystem. However, in Refs. 1 and 3 it was concluded that the determining factor is the structural phase transition.

It should be noted that superconductivity was detected at $13 \mathrm{~K}$ in thin FeTe films, deposited on special substrates that provide a strong deformation of the initial crystal structure. ${ }^{15}$ Assuming that the suppression of the structural and magnetic transitions can stimulate the appearance of superconductivity, attempts were made to create the SC state in bulk FeTe samples at high pressures. ${ }^{9,16}$ However, when measuring the resistivity of FeTe at pressures of up to $190 \mathrm{Kbar}$, there were no traces of SC. This is consistent with the observation of a negative pressure effect on the temperature of the transition to the superconducting state in tellurium enriched compounds $\mathrm{FeSe}_{1-x} \mathrm{Te}_{x}$ at $x \sim 0.8-0.9 .^{7}$ On the other hand, with increasing pressure, an unusual increase in FeTe magnetization was observed. ${ }^{16}$ Also, there was an anomalously large positive pressure effect on magnetic susceptibility in the paramagnetic and AFM phases of FeTe. ${ }^{14}$ Finally, it has recently been established ${ }^{17,18}$ that in FeTe there is a transition from AFM to the ferromagnetic phase at pressures above $2 \mathrm{GPa}$.

Thus, there are reasons to believe that magnetism of the FeTe compound cannot be explained by the appearance of spin-density waves that result from $(\pi, \pi)$ FS "nesting," similar to the "nesting" of electron and hole FS sheets in new iron-based superconductors. ${ }^{8}$ Assumptions are made ${ }^{12,19}$ that the observed bicollinear AFM ordering in FeTe can be due to the interaction of the localized spin moments, which includes input from delocalized electrons, without involving the "nesting" model. In Ref. 20, it is shown that doping FeTe with excess iron can lead to another type of "nesting" in $\mathrm{Fe}_{1+y} \mathrm{Te}-(\pi, 0)$, - which could explain the bicollinear AFM ordering (Fig. 1). However, this type of "nesting" was not confirmed by studies of FeTe using angle-resolved photoemission spectroscopy (ARPES). ${ }^{21}$

The totality of the aforementioned experimental and theoretical results points to the fact that electron and magnetic properties of $\mathrm{Fe}_{1+y} \mathrm{Te}$ compounds are determined by fine details of the crystal structure, and the amount of excess iron, $y$. Comprehensive studies of the electronic structure are necessary, in order to understand the mechanisms of magnetic ordering in FeTe, and to explain the occurrence of its magnetic and structural phase transitions. These studies allow us to shed light on the formation of superconducting states in compounds $\mathrm{FeSe}_{1-x} \mathrm{Te}_{x}$ and in particular, on the reasons for the absence of superconductivity in isostructural isovalent FeTe. In this study, we present the results of experimental and theoretical studies of the electronic structure of FeTe compounds in paramagnetic and antiferromagnetic states.

\section{Experimental details and results}

$\mathrm{Fe}_{1+y} \mathrm{Te}$ single crystals $(y \simeq 0,1)$ are grown using the methods described in Refs. 6 and 22. Typical dimensions of grown, plate-like single crystals were $1 \times 1 \times 0.2 \mathrm{~mm}$ (Fig. 2). The chemical composition of the samples was studied using a digital scanning electron microscope TESCAN Vega II XMU,

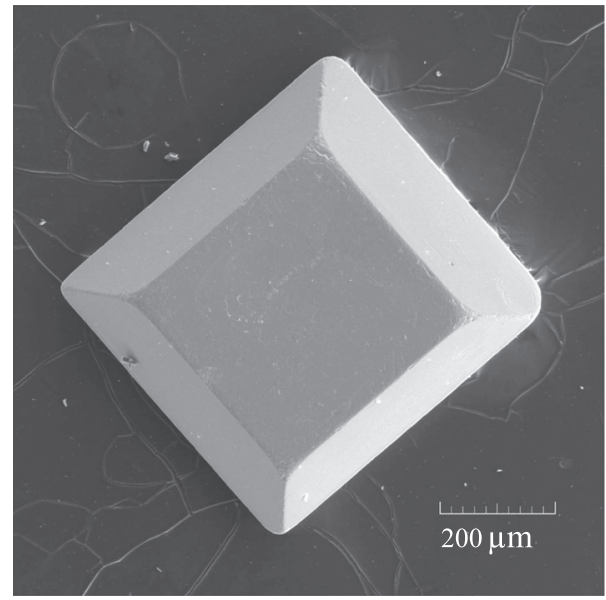

FIG. 2. A micrograph of a typical FeTe sample.

equipped with an energy dispersive microanalysis system INCA ENERGY 450.

$\mathrm{X}$-ray diffraction studies were conducted on an Xcalibur $S$ diffractometer, manufactured by Oxford Diffraction, with a two-dimensional CCD-detector at room temperature (graphite monochromator, Mo $K_{\alpha^{-}}$radiation, $293 \mathrm{~K}$ ). The processing of experimental data and accounting for absorption inside the crystal were done using the CrysAlis program, ${ }^{23}$ and the refinement of the structural model was done using the crystallographic software package JANA $2006 .^{24}$ Analysis of regular absorptions and symmetrically equivalent reflections allowed us to select space group $P 4 / \mathrm{nmm}$, which corresponds to the literature data for this compound. The atomic positional parameters from Ref. 25, are used as initial data to confirm the atomic structure. Using the refined structural data, the deformation electron density distribution was built for 66 of the structural amplitudes using $\sin \theta / \lambda$ $<0.6 \theta$

$$
\delta \rho(\mathbf{r})=\rho(\mathbf{r})-\sum_{i} \rho\left(\mathbf{r}-\mathbf{r}_{i}\right)
$$

wherein $\rho(\mathbf{r})$ is the electron density distribution in the crystal, and $\Sigma_{i} \rho\left(\mathbf{r}-\mathbf{r}_{i}\right)$ is the sum of the electron density distributions of spherically symmetric non-interacting atoms of this structure (Fig. 3, contour lines drawn through 0.02 elec$\left.\operatorname{tron} / \AA^{3}\right)$.

\section{Electronic structure of FeTe. Calculation results and analysis thereof}

In this paper, calculations of FeTe electronic structure were performed using a self-consistent method within the framework of the density functional theory (DFT), using a relativistic method of linearized MT (muffin-tin) orbitals with full potential (FP-LMTO), ${ }^{26-28}$ as well as augmented plane wave method with full potential (FP-LAPW), using the Elk software package. ${ }^{29}$ The exchange-correlation potential of conduction electrons was taken into account in the generalized gradient approximation (GGA) of the density functional theory. ${ }^{30}$ The DFT method with full potential is applicable to calculations of electron spectra of systems with a significant non-uniformity in electron density distribution. These systems include the FeTe compound investigated in this study. In the process of self-consistent calculation of 


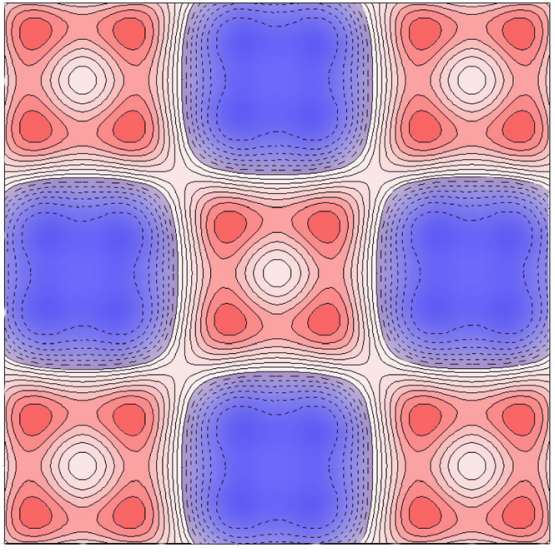

FIG. 3. The cross-section of deformation electron density distribution in the plane of iron atoms (001) for the paramagnetic phase of FeTe.

crystal potential, the spin-orbital interaction was taken into account at each iteration.

At temperatures above $70 \mathrm{~K}$ the compounds $\mathrm{Fe}_{1+y} \mathrm{Te}$ have a tetragonal crystal structure such as $\mathrm{PbO}$ (space group $P 4 / n m m$ ), in which the distance of the Te atoms from the plane of the iron atoms is determined by the internal structural parameter $Z$. As the temperature drops, at $T \simeq 70$, in $\mathrm{Fe}_{1+y} \mathrm{Te}(y \simeq 0.1)$ compounds there is a first order phase transition from the tetragonal to the monoclinic structure, which is accompanied by bicollinear AFM ordering. ${ }^{3,10,11}$ The corresponding parameters of the crystal structure of the paramagnetic and AFM phases of $\mathrm{Fe}_{1+y}$ Te were established in a series of studies using X-ray and neutron diffraction, ${ }^{2-4,10,25}$ and these experimental data are used in this article. For the bicollinear AFM phase, we chose the elementary magnetic cell $[2 a \times b \times c]$, corresponding to Fig. 1 . The structural parameters $a, b$, and $c$ were taken in accordance with Refs. 3 and 10, when the angle between the axes $a$ and $c$ was considered to be equal to $90^{\circ}$ (instead of $89.2^{\circ}$, for a monoclinic structure with low distortion).

The calculated main characteristics of the FeTe electronic structure in the paramagnetic phase are in a qualitative agreement with the results of previous calculations. $8,19,20,31,32$ According to the calculation of the electron density of states $N(E)$ in Fig. 4, it is in the paramagnetic phase that the FeTe compound has the highest density of states at the Fermi level among the systems $\mathrm{FeSe}_{1-x} \mathrm{Te}_{x}$, and the Stoner criterion is satisfied for the experimental values of the lattice parameters: $I N\left(E_{F}\right) \simeq 1$, where $I$ is the exchange interaction parameter. This points to the instability of the paramagnetic state of FeTe, and the possibility of transitioning to the ferromagnetic phase, which agrees with the recent observation of ferromagnetism in $\mathrm{Fe}_{1.03} \mathrm{Te}$ at pressures above $2 \mathrm{GPa} .{ }^{17,18}$ The Fermi surface for the paramagnetic phase, presented in Fig. 5, is composed of two corrugated cylinders, centered at the $\mathrm{M}$ point of the Brillouin zone, and also of three concentric hole sheets, surrounding the point of symmetry $\Gamma$, and is similar to the previously established FS for FeSe (see Refs. 8 and 34). In our comparison to the FS of FeSe, we should note the significantly large radii of quasi-cylindrical FS sheets, centered at the points of symmetry $\Gamma$ and $\mathrm{M}$.

The calculated partial electron densities of states in Fig. 4 indicate that in the vicinity of the Fermi level $E_{F}$, the input from the $d$-states of iron is a determining factor. Fig. 6 shows

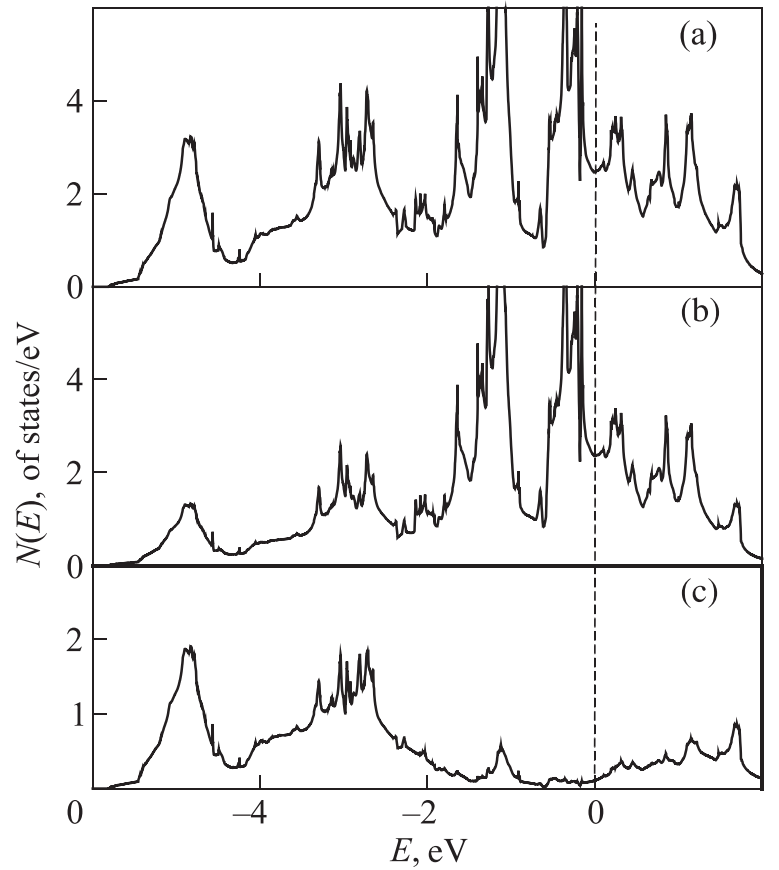

FIG. 4. The electron density of states $N(E)$ of the FeTe compound in the paramagnetic phase: (a) is the total density of states of FeTe per formula unit; (b) is the density of states of the iron atom; (c) is the density of states of the tellurium atom. The Fermi level $(E=0)$ is marked by a vertical line.

the distribution of the charge density in the basal plane (001) of the FeTe unit cell, as it was calculated in this study. The figure shows the four-flap electron density structure that typical for $d$-orbitals, and is in qualitative agreement with experimental data concerning the charge density distribution in Fig. 3. Note that the coordinate axes in Figs. 3 and 6 are rotated relative to each other by $45^{\circ}$. We should also take into account that the more spherical nature of the calculated electron density distribution in Fig. 6 is caused by averaging of the potential in the region of MT-spheres.

The calculations of equations $E_{\text {tot }}(V)$ for the magnetically ordered FeTe phases (ferromagnetic, collinear AFM, bicollinear AFM), completed in the GGA approximation, have shown that for the bicollinear AFM phase, the minimum total energy $E_{\text {tot }}$ is achieved, and that this phase is the ground state of the FeTe compound. The electron band structure, and spin-polarized electron density of states, are calculated for the ground state and shown in Figs. 7 and 8, respectively.

When there is antiferromagnetic ordering of FeTe, there is spin splitting in the density of states $N(E)$ (Figs. 8(b) and 8(c))

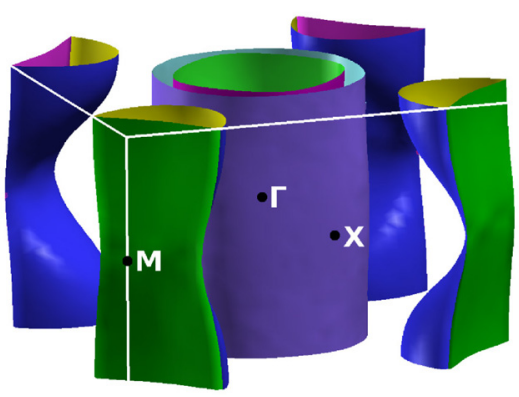

FIG. 5. The Fermi surface of the FeTe compound in the paramagnetic phase. Points of symmetry of the Brillouin zone are indicated according to Ref. 33. 


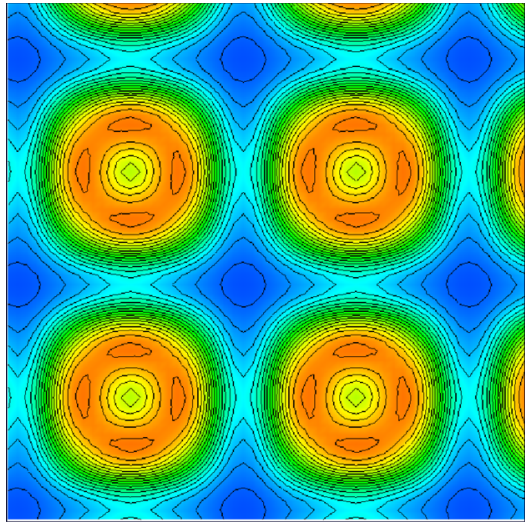

FIG. 6. The calculated contours of the charge density of the paramagnetic phase for the FeTe compound, in the iron atom plane (001).

and formation of magnetic moments on the iron atoms. The calculated value obtained in this article $M_{\mathrm{Fe}} \cong 2.37 \mu_{B}$ agrees with the neutron diffraction results $\left(M_{\mathrm{Fe}}^{\exp }=2.26-2.54 \mu_{B}\right.$ (Refs. 3 and 10)). Such a good agreement with experimental data serves as evidence of the fact that our band approach in the DFT-GGA approximation is adequate in studying the magnetic properties of FeTe. The calculated contours of the spin density in the (001) plane for the bicollinear antiferromagnetic phase of the FeTe compound are presented in Fig. 9.

Figure 10 shows the contours of the charge density distribution in the (100) plane of the FeTe unit cell, calculated in this article. The anisotropy of the charge density distribution between the iron atom and its nearest tellurium atom, is noticeable in the figure. We must note that the input from the $p$-states of Te into $N(E)$ of the FeTe valence band is significantly depleted (Fig. 8(d)) due to the charge transfer from Te to the neighboring $\mathrm{Fe}$ atoms, and the interstitial region. It can be assumed that the establishment of direct bonds between the neighboring atoms Fe and Te (Fig. 10) is caused by the hybridization of the $d$-orbitals of iron with $p$-orbitals of tellurium.

For a more detailed study of the nature of the chemical bonds in the FeTe compound, the crystal orbital overlap

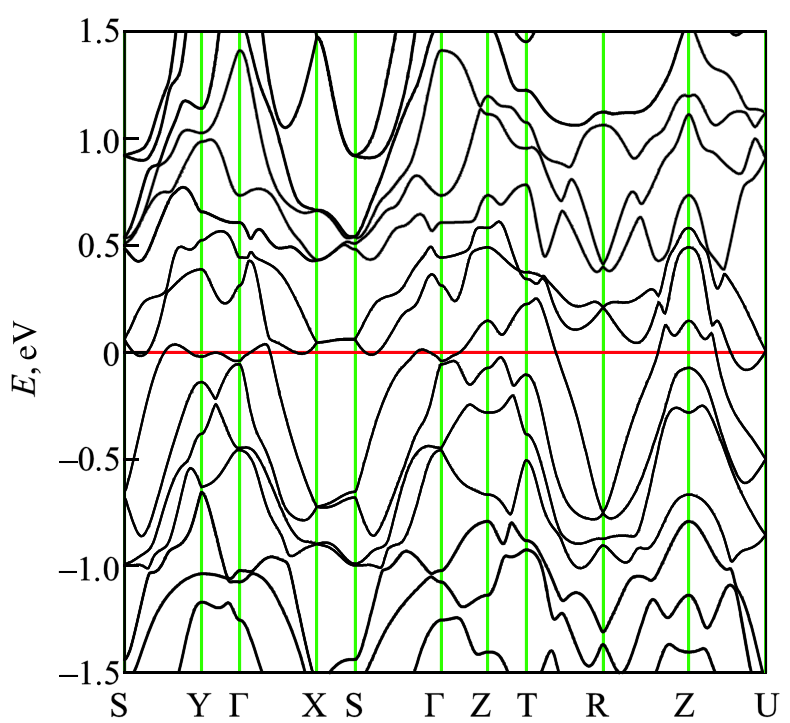

FIG. 7. Electron band structure of FeTe in the bicollinear AFM phase. The points of symmetry in the Brillouin zone are indicated in accordance with Ref. 33. The Fermi level is marked by the horizontal line $E=0$.

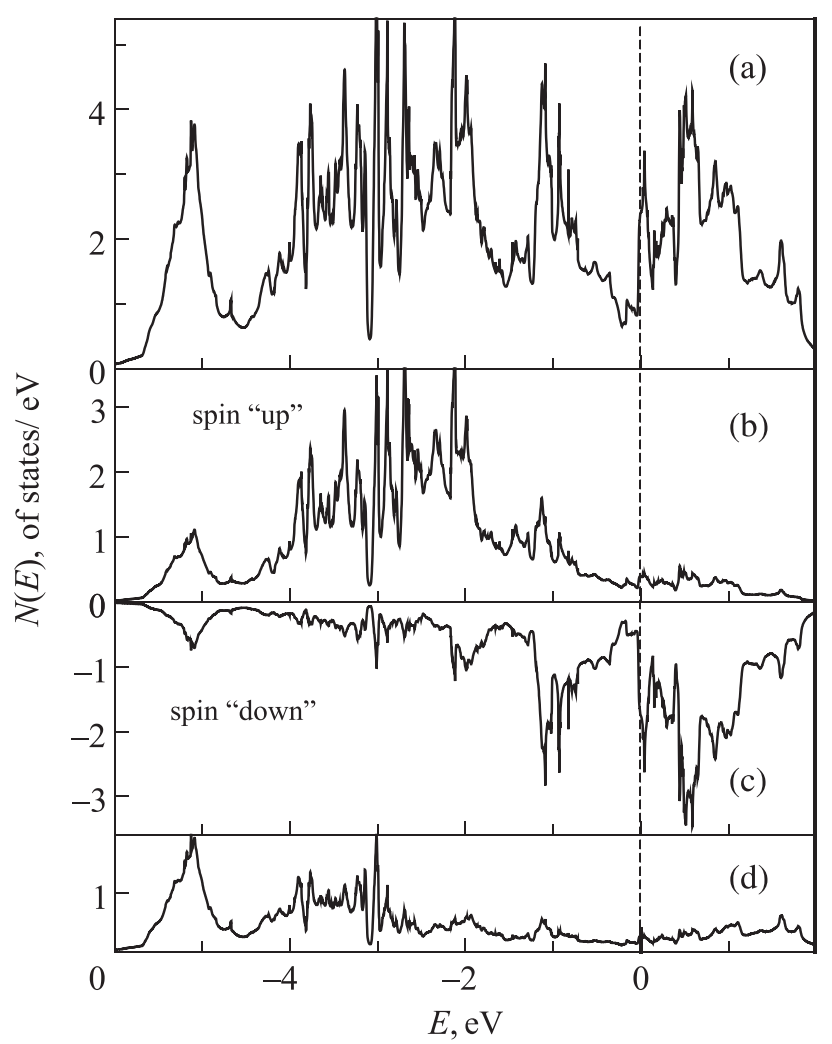

FIG. 8. Electron density of states $N(E)$ of the FeTe compound in the bicollinear antiferromagnetic phase: (a) total density of states for FeTe per formula unit; (b) spin-polarized density of states of the Fe atom (spin "up"); (c) spin-polarized density of states of the Fe atom (spin "down"); (d) density of states at the tellurium atom. Fermi level $(E=0)$ is marked by the vertical line.

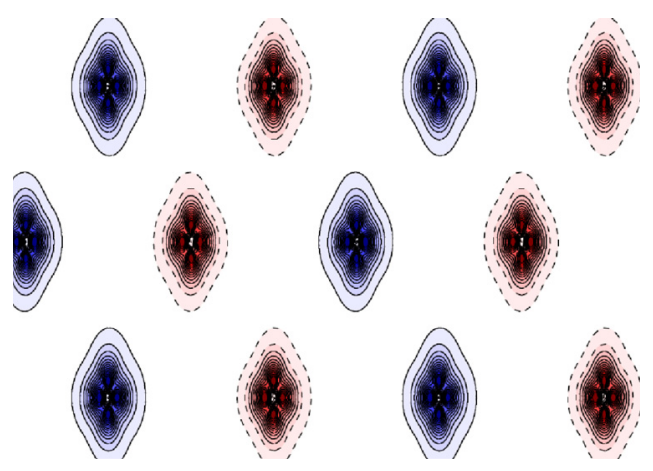

FIG. 9. Calculated contours of the spin density in the (001) plane for the bicollinear antiferromagnetic phase of the FeTe compound.

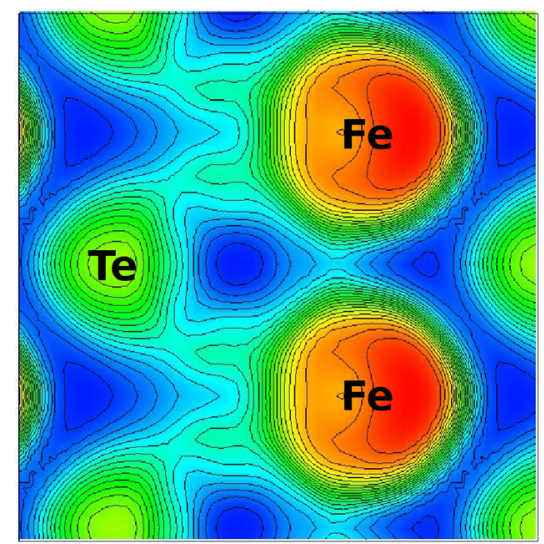

FIG. 10. Calculated contours of the charge density in the (100) plane for the FeTe compound in an antiferromagnetic phase. 
populations $\left(\mathrm{BCOOP}^{35}\right)$ are calculated in this study, within the framework for the FP-LMTO method. ${ }^{27}$ The calculated dependences $\operatorname{BCOOP}(E)$ (see Fig. 11) are a generalization of the known characteristics of molecule COOP (crystal orbital overlap population) for solids. The BCOOP values depend on the energy of the electron states of the valence band and, according to Ref. 35, turn out to be positive for the bonding orbitals, and negative for the antibonding orbitals and metallic bonds.

According to results of calculating $\operatorname{BCOOP}(E)$, the most pronounced bonding and antibonding states in FeTe form during the hybridization of the $p$-states of the closest tellurium atoms (Fig. 11(c)). Overlapping the Fe-Te orbitals enables bonding states near the bottom of the valence band (positive BCOOP $(E)$ in the interval $-4 \ldots-2 \mathrm{eV}$ in Fig. 11(b)). This agrees with the characteristic coincidence of energies belonging to the dominant regions of partial densities of iron $d$-states (Fig. 8(b)) and tellurium $p$-states (Fig. 8(d)) in the valence zone of FeTe. The bonding states are also realized when the $\mathrm{Fe}-\mathrm{Fe}$ orbitals are overlapped in the $-3 \ldots-1 \mathrm{eV}$ interval (Fig. 11(a)), which agrees with the calculation results for isovalent iron ruthenium ${ }^{35}$ Near the Fermi energy in the $-1 \ldots 1 \mathrm{eV}$ interval, for Fe-Te and Fe-Fe, we get negative values of $\operatorname{BCOOP}(E)$ (Figs. 11(a) and 11(b)), which point to a metal character in the chemical bond. The distribution of the electron density in the basal plane of FeTe (Figs. 3 and 6) is also typical for a metallic bond, whereas in the area between the planes $\mathrm{Fe}$ and Te, covalent bonds are clearly traceable (Fig. 10).

A sufficiently complex FS shape is established for the bicollinear AFM phase of the FeTe compound, as shown in Fig. 12. In this case, the Fermi surface of the low-temperature phase is radically different from the Fermi surface of paramagnetic FeTe in Fig. 5. This reconstruction of the FS at the AFM transition can be the reason for the changing sign of the Hall coefficient, as observed in FeTe. ${ }^{13,36}$ As a result of the multiband electronic structure of FeTe, there is a

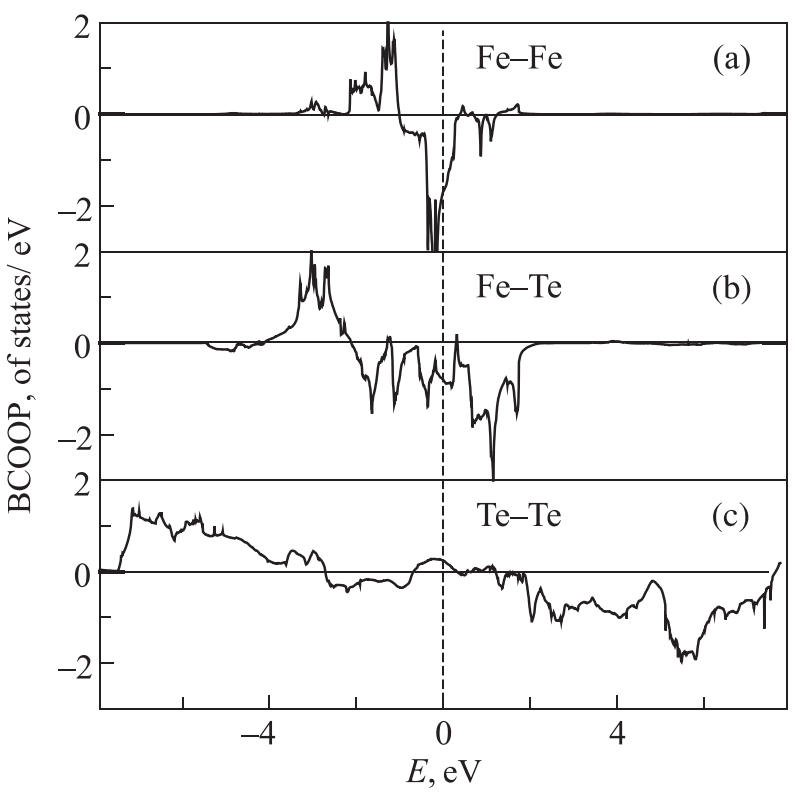

FIG. 11. The crystal orbital overlap population $\operatorname{BCOOP}(E)$ of the FeTe compound in the antiferromagnetic phase for the following pairs of nearest atoms in the unit cell: (a) Fe-Fe; (b) Fe-Te; (c) Te-Te. Fermi level $(E=0)$ is marked by a vertical line.

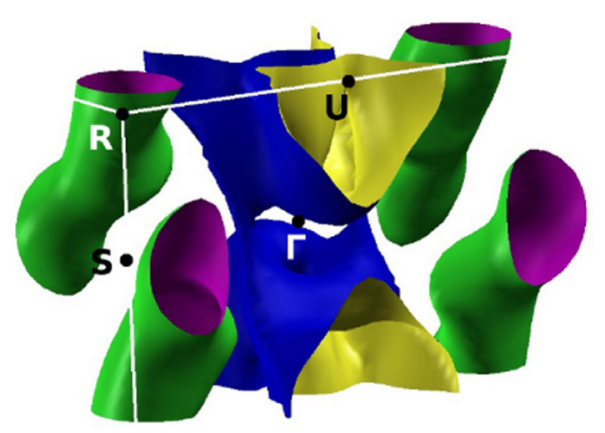

FIG. 12. Fermi surface of FeTe in a bicollinear AFM phase. Points of symmetry of Brillouin zone are indicated in accordance with Ref. 33.

redistribution of the inputs into the Hall coefficient from electron and hole sheets of the Fermi surface, at the AFM transition. It is necessary to note, however, that the quantitative analysis of the behavior of the Hall coefficient in FeTe would demand a detailed study of not only the FS, but also the changes in the rate of electron conduction and relaxation time.

The calculated value of the electron density of states for the AFM phase at the Fermi level $N\left(E_{F}\right) \simeq 3$ of states/eV, can be compared with the experimental data concerning the electron specific heat coefficient in FeTe, $\gamma_{\exp } \simeq 34 \mathrm{~mJ} /$ mol $\cdot K^{2}$ (Refs. 13 and 37 )

$$
\gamma_{\exp }=(1+\lambda) \gamma_{\text {theor }} \text {. }
$$

The corresponding renormalization parameter $\lambda$ includes the electron-phonon $\left(\lambda_{\mathrm{el}-\mathrm{ph}}\right)$ and spin-fluctuation $\left(\lambda_{s f}\right)$ inputs, and according to (2), comes to $\lambda \simeq 3$.8. It is necessary to note that the spin-fluctuation input $\lambda_{s f}$ can be sufficiently large for metallic systems close to magnetic instability. ${ }^{26}$ The sharp drop in the transition temperature to the superconducting state in $\mathrm{FeSe}_{1-x} \mathrm{Te}_{x}$ when approaching FeTe, indicates that strong spin-fluctuations at least do not contribute to the emergence of superconductivity in the FeTe system.

\section{Conclusion}

The results of this study indicate that the nature of the chemical bond in the FeTe compound is mainly metallic, and that it dominates in the basal plane of the iron atoms (001), with partially covalent components in the bonds between Fe-Te, Te-Te, and Fe-Fe. We can assume that the presence of covalent bonds contributes to the stabilization of the structural monoclinic distortions of the tetragonal phase of FeTe in the low-temperature region. Also, the hybridization of the iron $d$-states with telluride $p$-states leads to an expressed spatial anisotropy of the distribution of the charge density in the region between the planes of the $\mathrm{Fe}$ and $\mathrm{Te}$ atoms, and also to a charge transfer $\mathrm{Te} \rightarrow \mathrm{Fe}$.

In a number of studies the magnetism of the FeTe compound is interpreted within the framework of the localized momentum model at the iron atoms. ${ }^{12,17,38-40}$ The results of our calculations serve as evidence of the fact that the magnetic properties of FeTe are well-described within the framework of the delocalized electrons and DFT. In particular, it is found that the bicollinear AFM phase has a much lower total energy, than the paramagnetic, ferromagnetic, and collinear AFM phases, and is the ground state of the FeTe compound. The calculated value of the magnetic component 
of the bicollinear AFM phase $\left(M_{\mathrm{Fe}} \cong 2.4 \mu_{B}\right)$ is in good agreement with neutron diffraction data. The established fulfillment of the Stoner criterion $I N\left(E_{F}\right) \simeq 1$ for the paramagnetic state of FeTe in fact explains the found transition to the ferromagnetic state of the $\mathrm{Fe}_{1.03}$ Te system at pressures above 2 GPa. $^{18}$

In conclusion, it is necessary to note that there are serious problems in describing the magnetic properties of FeTe within the framework of the Heisenberg model, ${ }^{12,38,40}$ whereas taking into account the delocalized character of the $d$-electrons of iron allowed us to explain the observed variations of the magnetic anisotropy and AFM vector in compounds $\mathrm{Fe}_{1+y} \mathrm{Te}^{22}$

This study was supported by Grant RFFI NASU-02-14/ 78, and the computing resources of the B. Verkin ILTPE, NASU, Kharkiv grid cluster.

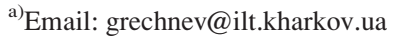

${ }^{1}$ T. M. McQueen, A. J. Williams, P. W. Stephens, J. Tao, Y. Zhu, V. Ksenofontov, F. Casper, C. Felser, and R. J. Cava, Phys. Rev. Lett. 103, 057002 (2009).

${ }^{2}$ Y. Mizuguchi and Y. Takano, J. Phys. Soc. Jpn. 79, 102001 (2010).

${ }^{3}$ A. Martinelli, A. Palenzona, M. Tropeano, C. Ferdeghini, M. Putti, M. R. Cimberle, T. D. Nguyen, M. Affronte, and C. Ritter, Phys. Rev. B 81, 094115 (2010).

${ }^{4}$ R. Viennois, E. Giannini, D. van der Marel, and R. Černý, J. Solid State Chem. 183, 769 (2010).

${ }^{5}$ A. V. Fedorchenko, G. E. Grechnev, V. A. Desnenko, A. S. Panfilov, S. L. Gnatchenko, V. V. Tsurkan, J. Deisenhofer, H.-A. Krug von Nidda, A. Loidl, D. A. Chareev, O. S. Volkova, and A. N. Vasiliev, Fiz. Nizk. Temp. 37, 100 (2011) [Low Temp. Phys. 37, 83 (2011)].

${ }^{6}$ Y. A. Ovchenkov, D. A. Chareev, E. S. Kozlyakova, O. S. Volkova, and A. N. Vasiliev, Physica C 489, 32 (2013).

${ }^{7}$ A. S. Panfilov, V. A. Pashchenko, G. E. Grechnev, V. A. Desnenko., A. V. Fedorchenko, A. N. Bludov, S. L. Gnatchenko, D. A. Chareev, E. S. Mitrofanova, and A. N. Vasilyev, Fiz. Nizk. Temp. 40, 793 (2014) [Low Temp. Phys. 40, 615 (2014)].

${ }^{8}$ A. Subedi, L. Zhang, D. J. Singh, and M. H. Du, Phys. Rev. B 78, 134514 (2008).

${ }^{9}$ Y. Mizuguchi, F. Tomioka, S. Tsuda, T. Yamaguchi, and Y. Takano, Physica C 469, 1027 (2009).

${ }^{10}$ S. Li, C. de la Cruz, Q. Huang, Y. Chen, J. W. Lynn, J. Hu, Y.-L. Huang, F.-C. Hsu, K.-W. Yeh, M.-K. Wu, and P. Dai, Phys. Rev. B 79, 054503 (2009).

${ }^{11}$ W. Bao, Y. Qiu, Q. Huang, M. A. Green, P. Zajdel, M. R. Fitzsimmons, M. Zhernenkov, S. Chang, M. Fang, B. Qian, E. K. Vehstedt, J. Yang, H. M. Pham, L. Spinu, and Z. Q. Mao, Phys. Rev. Lett. 102, 247001 (2009).

${ }^{12}$ F. Ma, W. Ji, J. Hu, Z.-Y. Lu, and T. Xiang, Phys. Rev. Lett. 102, 177003 (2009).

${ }^{13}$ G. F. Chen, Z. G. Chen, J. Dong, W. Z. Hu, G. Li, X. D. Zhang, P. Zheng, J. L. Luo, and N. L. Wang, Phys. Rev. B 79, 140509(R) (2009).

${ }^{14}$ A. V. Fedorchenko, G. E. Grechnev, V. A. Desnenko, A. S. Panfilov, S. L. Gnatchenko, V. Tsurkan, J. Deisenhofer, A. Loidl, O. S. Volkova, and A. N. Vasiliev, J. Phys.: Condens. Matter 23, 325701 (2011).
${ }^{15}$ Y. Han, W. Y. Li, L. X. Cao, X. Y. Wang, B. Xu, B. R. Zhao, Y. Q. Guo, and J. L. Yang, Phys. Rev. Lett. 104, 017003 (2010).

${ }^{16}$ H. Okada, H. Takahashi, Y. Mizuguchi, Y. Takano, and H. Takahashi, J. Phys. Soc. Jpn. 78, 083709 (2009).

${ }^{17}$ M. Bendele, A. Maisuradze, B. Roessli, S. N. Gvasaliya, E. Pomjakushina, S. Weyeneth, K. Conder, H. Keller, and R. Khasanov, Phys. Rev. B 87, 060409(R) (2013).

${ }^{18}$ M. Bendele, E. Pomjakushina, K. Conder, R. Khasanov, and H. Keller, J. Supercond. Nov. Magn. 27, 965 (2014).

${ }^{19}$ M. D. Johannes and I. I. Mazin, Phys. Rev. B 79, 220510(R) (2009).

${ }^{20}$ M.-J. Han and S. Y. Savrasov, Phys. Rev. Lett. 103, 067001 (2009).

${ }^{21}$ K. Nakayama, T. Sato, P. Richard, T. Kawahara, Y. Sekiba, T. Qian, G. F. Chen, J. L. Luo, N. L. Wang, H. Ding, and T. Takahashi, Phys. Rev. Lett. 105, 197001 (2010).

${ }^{22}$ G. E. Grechnev, A. S. Panfilov, A. V. Fedorchenko, A. A. Lyogenkaya, I. P. Zhuravleva, D. A. Chareev, A. N. Nekrasov, E. S. Mitrofanova, O. S. Volkova, A. N. Vasiliev, and O. Eriksson, J. Phys.: Condens. Matter 26, 436003 (2014).

${ }^{23}$ Crys AlisPro, Oxford Diffraction, Yarnton, Oxfordshire, England, 2011.

${ }^{24}$ V. Petriek, M. Dusek, and L. Palatinus, JANA 2006 (Institute of Physics, Prague, Czech Republic, 2006).

${ }^{25}$ I. O. Schichko, I. P. Makarova., M. Yu. Presnyakov, S. M. Kazakov, E. V. Antipov, D. A. Chareev, E. S. Mitrofanova, A. A. Mihutkin, and A. L. Vasilyev, Crystallography 60, 221 (2015) [Crystallogr. Rep. 60, 227 (2015)].

${ }^{26}$ G. E. Grechnev, Fiz. Nizk. Temp. 35, 812 (2009) [Low Temp. Phys. 35, 638 (2009)].

${ }^{27}$ J. M. Wills, M. Alouani, P. Andersson, A. Delin, O. Eriksson, and A. Grechnev, Full-Potential Electronic Structure Method. Energy and Force Calculations with Density Functional and Dynamical Mean Field Theory, Springer Series in Solid-State Sciences Vol. 167 (Springer-Verlag, Berlin, 2010).

${ }^{28}$ See http://fplmto-rspt.org/ for RSPt.

${ }^{29} \mathrm{http}$ ://elk.sourceforge.net/ for The Elk FP-LAPW Code.

${ }^{30}$ J. P. Perdew, K. Burke, and M. Ernzerhof, Phys. Rev. Lett. 77, 3865 (1996).

${ }^{31}$ M.-C. Ding, H.-Q. Lin, and Y.-Z. Zhang, Phys. Rev. B 87, 125129 (2013).

${ }^{32}$ A. Ciechan, M. J. Winiarski, and M. Samsel-Czekala, J. Phys.: Condens. Matter 26, 025702 (2014).

${ }^{33}$ C. Bradley and A. Cracknell, The Mathematical Theory of Symmetry in Solids: Representation Theory for Point Groups and Space Groups (Oxford University Press, Ely House, London, 1972).

${ }^{34}$ A. V. Logosha, G. E. Grechnev., A. A. Legenkaya, and A. S. Panfilov, Fiz. Nizk. Temp. 40, 405 (2014) [Low Temp. Phys. 40, 311 (2014)].

${ }^{35}$ A. Grechnev, R. Ahuja, and O. Eriksson, J. Phys.: Condens. Matter 15, 7751 (2003).

${ }^{36}$ Y. Liu, R. K. Kremer, and C. T. Lin, Supercond. Sci. Technol. 24, 035012 (2011).

${ }^{37}$ S. L. Bud'ko, P. C. Canfield, A. S. Sefat, B. C. Sales, M. A. McGuire, and D. Mandrus, Phys. Rev. B 80, 134523 (2009).

${ }^{38}$ C.-Y. Moon and H. J. Choi, Phys. Rev. Lett. 104, 057003 (2010).

${ }^{39}$ V. Gnezdilov, Yu. Pashkevich, P. Lemmens, A. Gusev, K. Lamonova, T. Shevtsova, I. Vitebskiy, O. Afanasiev, S. Gnatchenko, V. Tsurkan, J. Deisenhofer, and A. Loidl, Phys. Rev. B 83, 245127 (2011).

${ }^{40}$ J. K. Glasbrenner, J. P. Velev, and I. I. Mazin, Phys. Rev. B 89, 064509 (2014).

Translated by A. Bronskaya 